\title{
Surfactant and Water Ordering in Triacontanol Monolayers at the Water-Hexane Interface
}

\author{
Aleksey M. Tikhonov*,† and Mark L. Schlossman*," \\ Center for Advanced Radiation Sources, University of Chicago, Chicago, Illinois, National Synchrotron Light \\ Source, Beamline X19C, Brookhaven National Laboratory, Upton, New York 11973, and Departments of \\ Physics and Chemistry, University of Illinois at Chicago, 845 West Taylor Street, Chicago, Illinois 60607
}

Received: October 10, 2002; In Final Form: January 30, 2003

\begin{abstract}
Our view of molecular ordering in Langmuir monolayers at the water-vapor interface influences our understanding of molecular ordering at other interfaces, including liquid-liquid interfaces for which structural information is scarce. We present a comparative study of a monolayer of a long-chain alkanol at the watervapor and water-hexane interfaces using X-ray reflectivity to highlight significant differences between these two interfaces. The molecules in the Langmuir monolayer form an ordered phase of nearly rigid rods. In contrast, at the water-hexane interface, the triacontanol molecules form a condensed phase with progressive disordering of the chain from the $-\mathrm{CH}_{2} \mathrm{OH}$ to the $-\mathrm{CH}_{3}$ group. Surprisingly, at the water-hexane interface, the density in the headgroup region is $10-15 \%$ greater than either bulk water or the ordered headgroup region found at the water-vapor interface. It is conjectured that this higher density is a result of water penetration into the headgroup region of the disordered monolayer.
\end{abstract}

\section{Introduction}

Liquid-liquid interfaces play an important role in many chemical and biological systems. Water-oil interfaces are a model for the interaction of water with a hydrophobic molecular environment, important for protein folding and the formation of structures in complex fluids. Due to the experimental difficulties in directly probing the ordering of molecules at liquid-liquid interfaces, Langmuir monolayers consisting of organic molecules supported at the water-vapor interface have been often used to study the interfacial behavior of molecules of biological and technological relevance. Since these molecules often contain alkyl chains, a significant experimental and theoretical effort has been devoted to the study of long-chain surfactants containing a single alkyl chain such as fatty acids, alcohols, and esters. These molecules are known to form condensed phases at the water-vapor interface. ${ }^{1}$ Although these flexible chain molecules have gauche-trans chain disorder in bulk liquid phases, it is believed that the self-assembly of monolayers into closely packed structures tends to minimize the influence of gauche conformations. ${ }^{2}$ Except for molecular dynamics simulations, most of the theoretical work that accounts for the molecular structure in Langmuir monolayers assumes that the molecules behave as rigid rods. Phase diagrams consistent with many of the experimental results have been predicted from these rigid rod models. ${ }^{1}$

Although Langmuir monolayers are often used as models for molecular behavior at interfaces, there is a general expectation that molecules at a liquid-liquid interface are more disordered than at a liquid-vapor interface. It has been observed previously that the surface pressure, for a given molecular area, is usually larger at the water-oil interface than at the water-vapor interface. ${ }^{3}$ This has led to the belief that monolayers at the water-oil interface consist of molecules with disordered chains

\footnotetext{
* Corresponding author. E-mail: schloss@uic.edu; tikhonov@bnl.gov.

$\dagger$ University of Chicago and Brookhaven National Laboratory.

$\doteqdot$ Departments of Physics and Chemistry, University of Illinois at Chicago.
}

accompanied by extensive intermixing of the solvent into the monolayer. However, direct structural information on monolayers at the oil-water interface is scarce and has not supported the general expectation. Nonlinear optical studies have probed the ordering of short surfactants, sodium dodecylsulfonate, and sodium dodecylbenzenesulfonate (DBS), to demonstrate that the alkyl chain conformations are similar at both the water-vapor and water- $-\mathrm{CCl}_{4}$ interfaces, though the benzene rings in DBS orient differently at the two interfaces. ${ }^{4}$ Nonlinear optical studies of sodium dodecyl sulfate indicate a large degree of conformational disorder at both interfaces. ${ }^{5,6} \mathrm{X}$-ray reflectivity has been used to study partially fluorinated dodecanol (soluble in hexane) at the water-vapor and water-hexane interfaces. ${ }^{7,8}$ Again the chain ordering is similar at both interfaces; in this case the chain is rigid, and no solvent is mixed into the monolayer.

Here, we present a comparative study of a long-chain alkanol at the water-vapor and water-hexane interfaces. As expected, the alkanol monolayer spread at the water-vapor interface consists of nearly rigid rod molecules, though our highresolution X-ray data reveals a small disorder near the $-\mathrm{CH}_{3}$ end of the chain not previously measured. At the water-hexane interface, the same alkanol molecule exhibits a large and distinctive disorder likely due to a distribution of gauche defects along the chain with a progressively greater density of defects toward the $-\mathrm{CH}_{3}$ group near the bulk hexane. The data analysis indicates the presence of hexane molecules mixed into the region of the alkyl chain closer to the $-\mathrm{CH}_{2} \mathrm{OH}$ headgroup. In addition, the headgroup region has a density nearly $15 \%$ greater than bulk water, an effect not observed at the water-vapor interface.

\section{Monolayers at the Water-Vapor Interface}

The top curve in Figure 1A illustrates X-ray reflectivity data from a triacontanol $\left(\mathrm{CH}_{3}\left(\mathrm{CH}_{2}\right)_{29} \mathrm{OH}\right)$ monolayer spread at the water-vapor interface at a temperature $T=24^{\circ} \mathrm{C}$ and a surface pressure of $21.5 \pm 0.5 \mathrm{mN} / \mathrm{m}$ (X-ray kinematics is shown in Figure 1B). The monolayer was spread on a home-built Langmuir trough ${ }^{9}$ from a $2.1 \mathrm{mM}$ chloroform solution at a low 


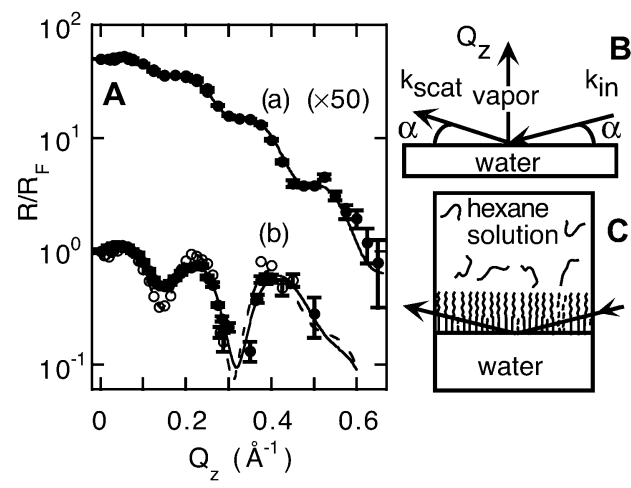

Figure 1. Normalized $\mathrm{X}$-ray reflectivity, $R / R_{\mathrm{F}}$, as a function of the wave vector transfer normal to the interface, $Q_{z}$, for triacontanol $\left(\mathrm{CH}_{3}\left(\mathrm{CH}_{2}\right)_{29} \mathrm{OH}\right)$ monolayers at the water-vapor and water-hexane interfaces. The X-ray reflectivity is normalized by the Fresnel reflectivity, $R_{\mathrm{F}}$, calculated for a structureless interface between two bulk media. (A) Top curve a is for triacontanol monolayers spread at the water-vapor interface, $T=24{ }^{\circ} \mathrm{C}$; curves $\mathrm{b}$ are for triacontanol monolayers adsorbed at the water-hexane interface, $T=24.07{ }^{\circ} \mathrm{C}$, open circles (fit is dashed line), or $T=24.52{ }^{\circ} \mathrm{C}$, filled circles (fit is solid line). Data for different temperatures were measured on different samples. Lines are fits described in the text. (B and C) X-ray kinematics. At the water-vapor interface, the X-rays travel through air then scatter off the surface. For the experiments on the water-hexane interface, the X-rays penetrate through the upper bulk phase of hexane solution, then scatter off a nearly planar water-hexane interface. The wave vector transfer, $\mathbf{Q}=\mathbf{k}_{\text {scat }}-\mathbf{k}_{\text {in }}$, is only in the $z$-direction normal to the interface, $Q_{z}=(4 \pi / \lambda) \sin \alpha$; therefore, only variations of the electron density normal to the interface are probed (the X-ray wavelength $\lambda=0.825 \pm$ $0.002 \AA$ for the water-hexane studies, $\lambda=1.5507 \pm 0.0002 \AA$ for the water-vapor studies, and $Q_{x}=Q_{y}=0$, where $x$ and $y$ are in the plane of the interface). X-ray reflectivity from the water-hexane interface was measured at beamline $\times 19 \mathrm{C}$ at the National Synchrotron Light Source (Brookhaven National Laboratory) with a liquid surface spectrometer and techniques specific to the liquid-liquid interface described in detail elsewhere. ${ }^{7,35}$ A similar spectrometer was used at the ChemMatCARS sector 15 at the Advanced Photon Source (Argonne National Laboratory) to study the monolayer of triacontanol at the water-vapor interface.

density (50 $\AA^{2} /$ molecule), then compression cycled eight times between surface pressures of $0 \mathrm{mN} / \mathrm{m}$ and $25 \mathrm{mN} / \mathrm{m}$ (with addition of pure chloroform at high pressures) to create a stable, homogeneous monolayer. The oscillations in the data are fitted by a standard procedure using the Born approximation along with a model for the interfacial profile that consists of three layers sandwiched between two bulk phases (bulk water and vapor). ${ }^{10}$ Two of these layers represent the electron density along the alkyl chain; the third layer represents the region of the $-\mathrm{CH}_{2}-$ $\mathrm{OH}$ groups. The interface between each of these layers and an adjacent layer (or bulk) is roughened by capillary waves with a roughness value similar to that calculated from capillary wave theory using the measured interfacial tension. ${ }^{11}$ The other fitting parameters include the electron density of each layer and the layer's thickness.

The resultant real space electron density profile of the monolayer at the water-vapor interface is shown in the top curve in Figure 2A. The overall thickness of the monolayer is $40 \pm 2 \AA$ and is nearly identical to the length of an all-trans triacontanol molecule, calculated to be $40.7 \AA .^{12-14}$ Most of the region of the monolayer corresponding to the alkyl chain has a normalized electron density of $1.014 \pm 0.003$ (normalized to the value for water of $0.333 \mathrm{e}^{-} / \AA^{3}$ ). This is comparable to literature values of 1.03 and 1.00 measured for the alkyl chain density in closely packed bulk phases of long chain alkanols $\left(23.3 \AA^{3} / \mathrm{CH}_{2}\right.$ and $24 \AA^{3} / \mathrm{CH}_{2}$, respectively $\left.{ }^{13}\right)$ and indicates that most of the chain is closely packed. The fit shown for the top

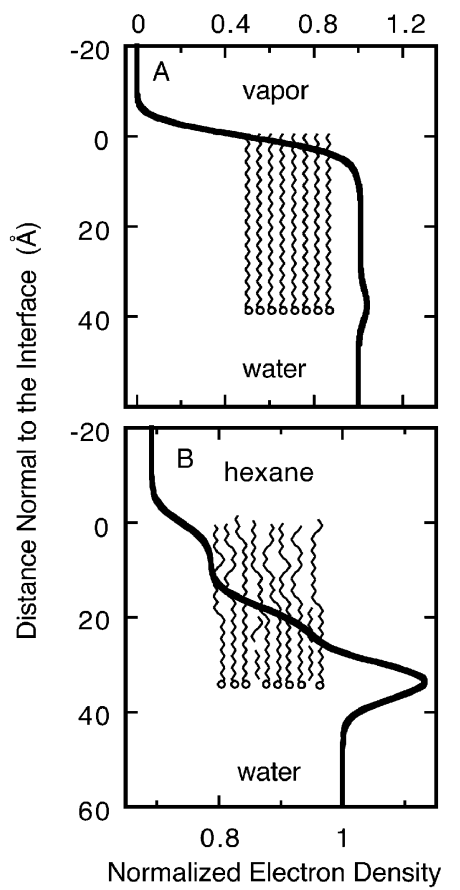

Figure 2. Electron density (normalized to the density for bulk water) as a function of the distance normal to the interface for a triacontanol monolayer at the water-vapor (A) and water-hexane (B) interfaces. Long molecules represent the triacontanol surfactants, and the short molecules in the water-hexane monolayer region represent hexane. The alkyl chains in the monolayer at the water-vapor interface are nearly closely packed, whereas those at the water-hexane interface are progressively disordered from a relatively ordered region near the water to a disordered liquidlike region adjacent to bulk hexane. Hexane is intermixed with the monolayer primarily near the headgroup region. The cartoon of molecules is for illustrative purposes only. The parameters for the data fitting describe the thickness and electron density for three layers sandwiched between bulk water and bulk hexane (or vapor). The electron densities are normalized to the value for bulk water $\left(0.333 \mathrm{e}^{-} / \AA^{3}\right)$. The normalized hexane density is 0.692 . Layer 1 is the headgroup region $\left(-\mathrm{CH}_{2} \mathrm{OH}\right)$, and layers 2 and 3 are for the alkyl tail group region $\left(-\left(\mathrm{CH}_{2}\right)_{28} \mathrm{CH}_{3}\right)$; layers are ordered water-1-2-3-hexane (or vapor); $L$ is the layer thickness; $\rho$ is the electron density. For the water-vapor interface: $L_{1}=5 \AA, \rho_{1, \max } / \rho_{\text {water }}=1.04 \pm 0.01, L_{2}=$ $24 \pm 5 \AA, \rho_{2} / \rho_{\text {water }}=1.014 \pm 0.003, L_{3}=11 \pm 4 \AA, \rho_{3} / \rho_{\text {water }}=$ $0.99(+0.01 /-0.04)$. For the water-hexane interface: $L_{1}=5 \AA$, $\rho_{1, \max } / \rho_{\text {water }}=1.13 \pm 0.01, L_{2}=13 \pm 2 \AA, \rho_{2} / \rho_{\text {water }}=0.95(+0.01 /-$ $0.02), L_{3}=18 \pm 1 \AA, \rho_{3} / \rho_{\text {water }}=0.79 \pm 0.01$. For the headgroup (layer 1) the maximum electron density is quoted rather than the density of the layer because the density and layer thickness fitting parameters are strongly correlated for this thin layer, but the resultant profile is well determined. The monolayer at the water-vapor interface is roughened by $3.25(+0.1 /-0.25) \AA$ similar to the calculated capillary roughness of $3.24 \AA$. At the water-hexane interface the layer is roughened by $3.6 \pm 0.3 \AA$ similar to the capillary wave value of 3.9 $\AA$. The total thickness of the monolayer at the water-vapor interface is $40 \pm 2 \AA$, at the water-hexane interface it is $36 \pm 2 \AA$. The alltrans length of triacontanol is $40.7 \AA$.

curve in Figure 1 requires a slightly lower electron density (0.99) toward the $-\mathrm{CH}_{3}$ group that we modeled by a third layer. This is consistent with molecular dynamics simulations that predict a small percentage of gauche conformations in these nearly rigid rod monolayers with the gauche defects concentrated near the $-\mathrm{CH}_{3}$ end. ${ }^{15,16}$

This analysis demonstrates that the triacontanol monolayer at the water-vapor interface is close packed with nearly alltrans and nearly upright molecules (normal to the interface). This is consistent with the understanding that molecules in the condensed phases of alkanol (and also alkanoic acid) Langmuir monolayers are nearly all-trans rigid rods. For example, X-ray 


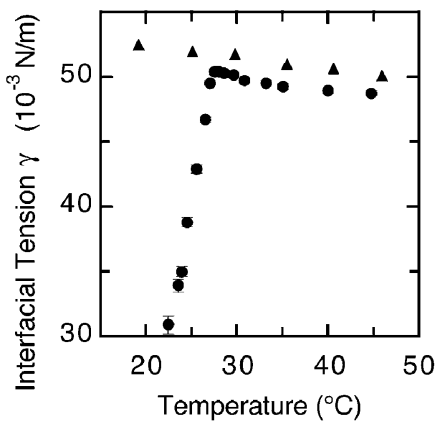

Figure 3. Interfacial tension as a function of temperature. Filled circles are for triacontanol monolayers adsorbed at the water-hexane interface. The tension of the pure water-hexane interface is shown (triangles) for comparison. Tensions were measured with a Wilhelmy plate in the sample cell used for X-ray scattering. ${ }^{36}$

surface diffraction studies of Langmuir monolayers of heneicosanol $\left(\mathrm{C}_{21} \mathrm{H}_{43} \mathrm{OH}\right)$ demonstrated that four ordered, closely packed phases are present over the temperature range of $14{ }^{\circ} \mathrm{C}<T<30{ }^{\circ} \mathrm{C}$ and surface pressures from 0 to $25 \mathrm{mN} /$ m. ${ }^{17,18}$ These four phases are distinguished by their lattice structures and chain tilt direction. In the highest pressure phase (for a surface pressure of approximately $20 \mathrm{mN} / \mathrm{m}$ and higher), the molecules are upright, ${ }^{17,18}$ similar to our measurement of triacontanol. Vibrational sum frequency spectroscopy studies of Langmuir monolayers of hexacosanoic acid $\left(\mathrm{C}_{26} \mathrm{H}_{53} \mathrm{O}_{2} \mathrm{H}\right)$ and hexadecanol $\left(\mathrm{C}_{16} \mathrm{H}_{33} \mathrm{OH}\right)$ indicated the absence of gauche conformations in the condensed phases, ${ }^{19,20}$ though IR reflection spectroscopy of stearyl alcohol $\left(\mathrm{C}_{18} \mathrm{H}_{43} \mathrm{OH}\right)$ and heneicosanol monolayers indicated the presence of some gauche conformers whose number decreases with increasing surface pressure. ${ }^{21,22}$

\section{Monolayers at the Water-Hexane Interface}

Monolayers at the water-hexane interface were prepared by placing a $0.7 \mathrm{mM}$ bulk solution of triacontanol in hexane on top of bulk water. Although insoluble in water, triacontanol is soluble in hexane, and the monolayer at the water-hexane interface can equilibrate by exchange of triacontanol between the interface and the bulk hexane. Figure 3 illustrates measurements of interfacial tension $\gamma$ as a function of temperature for triacontanol and for the pure water-hexane interface. The change $\Delta S$ in excess surface entropy across the transition is $4.3 \times 10^{-3} \mathrm{~J} / \mathrm{m}^{2} \mathrm{~K}$ (where $S=-\mathrm{d} \gamma / \mathrm{d} T$ and $\Delta S$ is given by the slope difference on either side of the kink at $T=27.5^{\circ} \mathrm{C}$ ). This is larger than $\Delta S=1.3 \times 10^{-3} \mathrm{~J} / \mathrm{m}^{2} \mathrm{~K}$ measured previously for surface freezing at a pure $\mathrm{C}_{28}$ alkanol-vapor interface (the quoted value is half that for freezing of a bilayer). ${ }^{23}$ It is reasonable to expect a much larger $\Delta S$ in our system since the transition occurs when alkanol molecules in a dilute bulk solution form a condensed monolayer at the interface, whereas surface freezing occurs when the top two layers of molecules at a liquid alkanol surface freeze into a solid bilayer. The thermodynamic measurement in Figure 3 indicates that the triacontanol monolayer undergoes a single transition from a low temperature ordered phase to a high-temperature disordered phase similar to thermodynamic measurements from shorter alkanols at the water-hexane interface. ${ }^{24-26}$

We chose to study the monolayer at temperatures $(T=24.07$ ${ }^{\circ} \mathrm{C}$ and $24.52{ }^{\circ} \mathrm{C}$ ) slightly above the temperature at which the bulk hexane is saturated with triacontanol (as observed by the formation of triacontanol crystallites at $T \approx 22^{\circ} \mathrm{C}$ ). Under these conditions, the triacontanol monolayer at the water-hexane interface will be close to its densest state for that temperature. $\mathrm{X}$-ray reflectivity is used to directly probe this interface in the geometry illustrated in Figure 1C. ${ }^{12}$ Reflectivity data are shown in the bottom curves in Figure 1A. The fits shown for these data are performed in the same way as for the monolayer at the water-vapor interface. The electron density interfacial profile that results from the fitting procedure for the data measured at $T=24.52{ }^{\circ} \mathrm{C}$ is shown in Figure $2 \mathrm{~B}$, a similar profile is obtained for the sample at $T=24.07^{\circ} \mathrm{C}$.

Comparison of panels $\mathrm{A}$ and $\mathrm{B}$ of Figure 2 indicates that the molecular ordering at the water-hexane interface is very different from that at the water-vapor interface. Again, two layers are required to model the alkyl chain (plus one layer for the headgroup) in order to fit the data. However, the normalized electron densities for the two layers modeling the chain are 0.95 \pm 0.01 and $0.79 \pm 0.01$, both different from the closely packed value of 1.03 (or 1.00). The normalized electron density of 0.79 near the $-\mathrm{CH}_{3}$ group corresponds closely to the value of 0.81 for bulk liquid alkyl chains near their freezing point (bulk measurements yield $\left.29.6 \AA^{3} / \mathrm{CH}_{2}\right) .{ }^{13}$ In the bulk it is believed that liquid $n$-alkanes near the freezing point are arranged in quasilamellar regions with gauche conformations to cause chain disorder, while still maintaining a good alignment of neighboring molecules. ${ }^{27,28}$ Although not unambiguously proven, it seems that a similar arrangement occurs in the part of the triacontanol chain near the $-\mathrm{CH}_{3}$. A disordered liquidlike part of the chain would occupy a surface area of at least $23.4 \AA^{2} /$ chain, an increase of $\sim 25 \%$ over the closely packed area/molecule of 18.5 $\AA^{2} /$ chain. In the region of the alkyl chain close to the $-\mathrm{CH}_{2}$ $\mathrm{OH}$ group, the chain has to be more ordered to yield a normalized electron density of 0.95 . In addition, either water or hexane must be mixed into this part of the monolayer to properly account for the available volume in the monolayer region. A compelling arrangement is a well-ordered chain near the $-\mathrm{CH}_{2} \mathrm{OH}$ group that is mixed with hexane $(20-25 \mathrm{vol} \%)$ and a progressively more disordered chain toward the $-\mathrm{CH}_{3}$ end of the tail group (as illustrated in Figure 2B). This arrangement is quantitatively consistent with our measured densities.

The disorder in the chain will account, at least partially, for the overall monolayer thickness $(36 \pm 2 \AA$ ) being less than the length of an all-trans triacontanol molecule (40.7 $\AA$ ). For example, a single kink defect (gtg' or $\mathrm{g}^{\prime}$ tg conformation) will maintain the overall chain orientation while reducing its length by $0.6 \AA$ to $0.7 \AA$. In addition, the triacontanol molecules may be tilted slightly from the normal to the interface, but the reflectivity does not directly probe this tilt. Although grazing incidence diffraction could directly probe this tilt if the monolayer was sufficiently ordered, the background scattering from the bulk precludes its measurement.

\section{Discussion}

The electron density in the headgroup region $\left(-\mathrm{CH}_{2} \mathrm{OH}\right)$ is larger at the water-hexane interface $\left(\rho_{\max }=1.13 \pm 0.01\right)$ than at the water-vapor interface $\left(\rho_{\max }=1.04 \pm 0.01\right.$, with $\rho_{\text {bulk }}$ water $\equiv 1)$. Since the area per headgroup is larger at the waterhexane interface due to disorder in the monolayer, the additional electron density cannot be attributed to closer packing of headgroups. In addition, the higher density is not likely due to the interaction of water with hexane since X-ray measurements of the pure water-hexane interface do not reveal an enhanced interfacial density of water. ${ }^{29}$ The lower density of headgroups at the water-hexane interface may allow for water penetration into the headgroup region, which then induces a higher density in this region.

We suggest that a mechanism to produce the higher density is orientational ordering of penetrated water by the polar $-\mathrm{CH}_{2-}$ 
$\mathrm{OH}$. Interfacial electric fields can align interfacial water. For example, orientational ordering of interfacial water at a charged interface has been inferred or observed in several experiments. ${ }^{30-32}$ It has been recently proposed from experiments and molecular dynamics simulations that water in the first hydration shell of lysozyme and other proteins has an average density approximately $10-20 \%$ greater than the bulk density, similar in magnitude to the change we observed, and that this higher density is due to orientational ordering of water molecules in depressions on the protein surface. ${ }^{33,34}$

We have also measured X-ray reflectivity from triacontanol at the water-hexane interface for higher temperatures through the region of the transition indicated in Figure 3 up to $T=45$ ${ }^{\circ} \mathrm{C}$ (data not shown). At temperatures above the transition, the triacontanol molecules have mostly desorbed from the interface into the bulk hexane solution. Near the transition temperature $\left(T=27.5^{\circ} \mathrm{C}\right)$, a sharp change in the reflectivity, accompanied by additional X-ray diffuse scattering above that expected from capillary waves, is consistent with the presence of domains of triacontanol separated by regions of essentially pure waterhexane interface (gaseous monolayer regions). This is similar to X-ray measurements from fluorinated monolayers at the water-hexane interface which also undergo an order-disorder transition. For that system, X-ray diffuse scattering and reflectivity revealed a sharp transition in temperature from a low temperature interface that is fully covered by a condensed monolayer to a dilute high-temperature phase. ${ }^{8}$ The temperature variation of the reflectivity for the triacontanol system is similar to the fluorinated system and indicates that at low temperatures $\left(T \approx 24^{\circ} \mathrm{C}\right.$, as for the data in Figure 1$)$ the nearly fully covered interface may contain a small fraction $(\sim 10 \%)$ of gaseous regions. If present, these gaseous regions will not affect our qualitative conclusions regarding the molecular ordering of triacontanol, but will quantitatively modify them slightly.

Our conclusion regarding a progressive distribution of gauche conformations along the alkyl chain that increases away from the $-\mathrm{CH}_{2} \mathrm{OH}$ group is sensible, considering the constraints of placing a headgroup at the water-hexane interface and orienting the alkyl chain toward the hexane. A similar effect was observed, though to a lesser extent, in molecular dynamics simulations of Langmuir monolayers in which there are a small number of gauche conformations that appear primarily at the chain ends. ${ }^{1,15}$ Also, in the liquid phase of bulk alkanols far from the freezing point, NMR experiments have shown that a seven-carbon-long region of the alkyl chain near the $-\mathrm{CH}_{2} \mathrm{OH}$ group of 1-dodecanol $\left(\mathrm{CH}_{3}\left(\mathrm{CH}_{2}\right)_{11} \mathrm{OH}\right)$ has a constant degree of order with increasing chain disorder further out along the chain. ${ }^{13}$ In the bulk liquid, hydrogen bonding between nearest neighbor $-\mathrm{CH}_{2}-$ $\mathrm{OH}$ groups provides the constraint that establishes the pattern of chain ordering. This is a weaker constraint than that provided by a flat water-hexane interface.

These studies demonstrate that long-chain alkanols that form rigid-rod monolayers at the water-vapor interface instead form monolayers at the water-hexane interface with a well defined disorder. The molecular ordering is characterized by chain disorder that progressively varies from relatively well-ordered chains near the $-\mathrm{CH}_{2} \mathrm{OH}$ group to liquidlike chain densities at the $-\mathrm{CH}_{3}$ end of the molecule. Solvent mixing into the monolayer accompanies this chain disorder. We conjecture that ordering of water molecules that have penetrated into the region of the headgroup will account for the high density in this region. These results have important implications for understanding water structuring near biological macromolecules and alkyl chain ordering in many systems.
Acknowledgment. We acknowledge the valuable assistance of Binhua Lin (U of Chicago), Guangming Luo (UIC), Sai Venkatesh Pingali (UIC), Mati Meron (U of Chicago), Tim Graber (U of Chicago), David Schultz (UIC), and Jeff Gebhardt (U of Chicago) at the APS. Financial support is gratefully acknowledged from NSF DMR (for M.L.S.), NSF Chemistry (for ChemMatCARS), and DOE (for ChemMatCARS, Brookhaven, and Argonne National Laboratories).

\section{References and Notes}

(1) Kaganer, V. M.; Mohwald, H.; Dutta, P. Rev. Mod. Phys. 1999, 71,779

(2) Shin, S.; Rice, S. A. Langmuir 1994, 10, 262.

(3) Davies, J. T.; Rideal, E. K. Interfacial Phenomena; Academic Press: New York, 1961.

(4) Watry, M.; Richmond, G. L. J. Am. Chem. Soc. 2000, 122, 875.

(5) Bell, G. R.; Bain, C. D.; Ward, R. N. J. Chem. Soc., Faraday Trans. 1996, 92,515 .

(6) Conboy, J. C.; Messmer, M. C.; Richmond, G. L. J. Phys. Chem. 1996, $100,7617$.

(7) Zhang, Z.; Mitrinovic, D. M.; Williams, S. M.; Huang, Z.; Schlossman, M. L. J. Chem. Phys. 1999, 110, 7421.

(8) Tikhonov, A. M.; Li, M.; Mitrinovic, D. M.; Schlossman, M. L. J. Phys. Chem. B 2001, 105, 8065 .

(9) Acero, A. A.; Li, M.; Lin, B.; Rice, S. A.; Goldman, M.; Azouz, I. B.; Goudot, A.; Rondelez, F. J. Chem. Phys. 1993, 99, 7214.

(10) Tidswell, I. M.; Ocko, B. M.; Pershan, P. S.; Wasserman, S. R.; Whitesides, G. M.; Axe, J. D. Phys. Rev. B 1990, 41, 1111.

(11) Mitrinovic, D. M.; Tikhonov, A. M.; Li, M.; Huang, Z.; Schlossman, M. L. Phys. Rev. Lett. 2000, 85, 582.

(12) For water-hexane interface studies, the liquid samples are stirred and equilibrated in a temperature-controlled, vapor-tight stainless stee sample cell described in detail elsewhere (see ref 7). Hexane was purified by passing it through a column of activated alumina several times; triacontanol was purified by double crystallization from the purified hexane; water was from a Barnstead Nanopure system.

(13) Small, D. M. The Physical Chemistry of Lipids; Plenum: New York, 1986.

(14) Israelachvili, J. N. Intermolecular and Surface Forces; Academic Press: London, England, 1992

(15) Harris, J.; Rice, S. A. J. Chem. Phys. 1988, 89, 5898

(16) Bareman, J. P.; Cardini, G.; Klein, M. L. Phys. Rev. Lett. 1988 $60,2152$.

(17) Lin, B. Ph.D. Thesis, Northwestern University, Evanston, IL, 1990.

(18) Shih, M. C.; Bohanon, T. M.; Mikrut, J. M.; Zschack, P.; Dutta, P. J. Chem. Phys. 1992, 97, 4485

(19) Miranda, P. B.; Du, Q.; Shen, Y. R. Chem. Phys. Lett. 1998, 286,1

(20) Wolfrum, K.; Laubereau, A. Chem. Phys. Lett. 1994, 228, 83.

(21) Buontempo, J. T.; Rice, S. A. J. Chem. Phys. 1993, 99, 7030.

(22) Buontempo, J. T.; Rice, S. A. J. Chem. Phys. 1993, 98, 5835.

(23) Ocko, B. M.; Wu, X. Z.; Sirota, E. B.; Sinha, S. K.; Gang, O.; Deutsch, M. Phys. Rev. E 1997, 55, 3164.

(24) Matubayasi, N.; Motomura, K.; Aratono, M.; Matuura, R. Bull. Chem. Soc. Jpn. 1978, 51, 2800.

(25) Lin, M.; Firpo, J.-L.; Mansoura, P.; Baret, J. F. J. Chem. Phys. 1979, 71, 2202.

(26) Tikhonov, A. M.; Li, M.; Schlossman, M. L. BNL National Synchrotron Light Source Activity Report 2001 (http://nslsweb.nsls.bnl.gov/ nsls/pubs/actrpt/2001/sec2_scihi_softmat_tikhonov.pdf, accessed 2002).

(27) Stewart, G. W.; Morrow, R. M. Phys. Rev. 1927, 30, 232.

(28) Brady, G. W.; Fein, D. B. J. Appl. Crystallogr. 1975, 8, 261.

(29) Mitrinovic, D. M.; Zhang, Z.; Williams, S. M.; Huang, Z.; Schlossman, M. L. J. Phys. Chem. B 1999, 103, 1779.

(30) Toney, M. F.; Howard, J. N.; Richer, J.; Borges, G. L.; Gordon, J. G.; Melroy, O. R.; Wiesler, D. G.; Yee, D.; Sorenson, L. B. Nature 1994, $368,444$.

(31) Gragson, D. E.; McCarty, B. M.; Richmond, G. L. J. Phys. Chem. 1996, 100,14272

(32) Richmond, G. L. Chem. Rev. 2002, 12, 2693.

(33) Svergun, D. I.; Richard, S.; Koch, M. H. J.; Sayers, Z.; Kuprin, S.; Zaccai, G. Proc. Natl. Acad. Sci. U.S.A. 1998, 95, 2267.

(34) Merzel, F.; Smith, J. C. Proc. Natl. Acad. Sci. U.S.A. 2002, 99 , 5378.

(35) Mitrinovic, D. M.; Williams, S. M.; Schlossman, M. L. Phys. Rev. E 2001, 63, 021601.

(36) Adamson, A. W. Physical Chemistry of Surfaces, 5th ed.; John Wiley \& Sons: New York, 1990. 